\title{
INFERÊNCIA DO DIAGNÓSTICO DE ENFERMAGEM DOR AGUDA E O PERFIL DE PACIENTES NÃO COMUNICATIVOS DE UM HOSPITAL UNIVERSITÁRIO
}

\author{
Marisa Dibbern Lopes Correia ${ }^{1}$ \\ Juliana Prado Biani Manzoli ${ }^{2}$ \\ Ráisa Camilo Ferreira ${ }^{3}$ \\ Luciana Aparecida Costa Carvalho ${ }^{4}$ \\ Paula Cristina Pereira da Costa ${ }^{5}$ \\ Micneias Lacerda Botelho ${ }^{6}$ \\ Elaine Ribeiro ${ }^{7}$ \\ Fábio Luis Montanari ${ }^{8}$ \\ Natanaellin Eydiane da Silva Begnami ${ }^{9}$ \\ Bruna Valentina Zuchatt ${ }^{10}$ \\ Erika Christiane Marocco Duran ${ }^{11}$ \\ 1,3,5,7 Mestre em Enfermagem. Doutoranda pela Faculdade de Enfermagem da Unicamp. \\ 2,4,9,10 Mestranda em Enfermagem pela Unicamp. \\ ${ }^{6}$ Mestre em Educação. Doutorando pela Faculdade de Enfermagem da Unicamp. \\ ${ }^{8}$ Enfermeiro assistencial do HC Unicamp. \\ ${ }^{11}$ Professora Doutora da Faculdade de Enfermagem Unicamp.
}

INTRODUÇÃO: A dor em pacientes criticamente enfermos é de difícil identificação mas a observação de indicadores clínicos nesses pacientes, por enfermeiros, poderá auxiliar na identificação precoce do Diagnóstico de Enfermagem (DE) Dor Aguda e assim, melhorar a assistência prestada. OBJETIVO: descrever o perfil de pacientes não comunicativos e a presença do DE Dor Aguda e seus componentes. MÉTODO: Estudo longitudinal conduzido de janeiro a julho de 2018 em Unidade de Terapia Intensiva de Hospital Universitário, com 96 pacientes não comunicativos em repouso (T0), em procedimento não doloroso (checagem do pulso radial) (T1) e doloroso (mudança de decúbito) (T2). Foram coletados dados clínicos, aplicada a escala de coma de Glasgow nos pacientes não sedados e Ramsay nos sedados, além de avaliados os componentes do DE Dor Aguda em cada tempo, por dois avaliadores. A concordância interobservador foi analisada por meio do teste Kappa. Parecer 1950820 do Comitê de Ética da Unicamp. RESULTADOS: Dos 96 pacientes, 64,6\% ( $n=62$ ) eram homens, tinham idade média de 53,5 anos (DP $16,6)$ e a não comunicação se deu pelo tubo orotraqueal $(89,6 \% n=86)$ e sedação $(46,9 \% n=45)$. A maioria era pacientes clínicos $(61,5 \% n=59)$, tiveram escore 3 na escala de Glasgow $(36,2 \% \mathrm{n}=17)$ ou escore 6 na escala de Ramsay ( $83,7 \%$ $n=41)$. Recebiam fentanil $(59,4 \% n=57)$ e $60,4 \%(n=58)$ tinham pelo menos um 
analgésico prescrito. As características definidoras (CD) do DE Dor Aguda em T0 foram: diaforese $(n=7)$, expressão facial $(n=6)$ e evidência de dor usando lista de verificação $(n=2)$ e um paciente recebeu o DE Dor Aguda. No T2 foram: evidência de dor usando lista de verificação $(n=22)$, expressão facial $(n=18)$, diaforese $(n=8)$, mudança de parâmetros fisiológicos $(n=7)$, dilatação pupilar $(n=1)$ e 24 pacientes receberam o $D E$. Os fatores relacionados foram os agentes lesivos biológicos $(n=76)$ e físicos $(n=59)$. O Kappa apontou moderada a alta concordância $(0,48-1,00)$ interobservador. CONCLUSÃO: a principal causa da não comunicação foi a presença do tubo orotraqueal e a maioria dos pacientes recebia analgésicos. O procedimento doloroso influenciou no aumento da ocorrência do DE Dor Aguda e de suas CD, sendo as mais marcantes a evidência de dor usando lista de verificação seguida pela expressão facial. Os avaliadores apresentaram boa concordância na avaliação dos pacientes.

Palavras-chave: Enfermagem. Diagnóstico de enfermagem. Dor aguda. 